\title{
La motivación para aprender inglés en estudiantes de licenciatura, un estudio empírico en México
}

\section{Undergraduate Students' Motivation to learn English, an Empirical Study in Mexico}

\author{
Jaime U. Ramírez-Vega ${ }^{a}$
}

\begin{abstract}
:
This article reports the first results of a standardized-test application phase of a larger-scale study that was carried out with high school students. This larger-scale study aims to describe motivation with the L2 Motivational Self System (Dörnyei, 2009) in more than 600 high school students. However, this article shows the results of the questionnaire administered to 57 students of the Bachelor of Design and Visual Communication of the Faculty of Arts and Design, UNAM where the subject of English is compulsory and curricular; The items were designed under the 6-point Likert scale with 67 questions for motivation and academic profile. The data obtained provide a solid empirical basis for the characteristics of English learning when it is learned curricular and compulsory. Likewise, the results of this study suggest a relationship between motivation, teacher influence and class experience.
\end{abstract}

Keywords:

learning, English, EFL, ESL, motivation, L2 Motivational System, undergraduate students

\section{Resumen:}

El presente artículo muestra los resultados de la última fase de piloto implementada de un estudio a mayor escala que se llevó a cabo con alumnado de bachillerato. El estudio a gran escala pretende describir la motivación con el Sistema Motivacional del Yo (Dörnyei, 2009) en más de 600 estudiantes de bachillerato. No obstante, en este artículo se muestran los resultados del cuestionario sobre la motivación en su fase piloto administrado a 57 estudiantes de la licenciatura de Diseño y Comunicación Visual de la Facultad de Artes y Diseño de la UNAM donde la asignatura de inglés es obligatoria y curricular; los reactivos fueron diseñados bajo la escala Likert a 6 puntos con 67 preguntas para la motivación y su perfil académico. Los datos obtenidos proveen una base sólida empírica de las características del aprendizaje del inglés en esta muestra cuando éste se aprende en un contexto donde el idioma es curricular y obligatorio. Asimismo, los resultados de este estudio dejan entrever que existe una relación entre la motivación, la influencia del profesorado, sus estrategias didácticas y la experiencia de clase como factores clave.

\section{Palabras Clave:}

ESL, Sistema Motivacional del Yo, licenciatura, lengua, inglés, aprendizaje, motivación, EFL.

\section{INTRODUCCIÓN}

La motivación para aprender una lengua extranjera ha sido uno de los temas relevantes en las últimas décadas (Bursalı1 y Öz, 2017). Algunos la consideran multifactorial. A partir del grado de motivación, las personas deciden el tiempo que dedican a estudiar una lengua extranjera por lo que se ha puesto relevancia en el aprendizaje del inglés desde las últimas décadas. La motivación puede estar influida por variables externas como las políticas educativas, los cambios curriculares, políticas migratorias, políticas de los países que hablan estas lenguas, por mencionar algunos; asimismo, por variables más relacionadas con el contexto del estudiantado como el entorno de la institución educativa, la práctica docente, las amistades, los familiares, el interés por la cultura del otro, así como variables individuales (gustos e intereses propios).

Jaime Ulises Ramírez Vega, Universidad Nacional Autónoma de México, https://orcid.org/0000-0003-0541-9030, Email: jaime.ramirez@enp.unam.mx 
Existen estudios que muestran una relación significativa entre los logros de aprendizaje y la motivación por aprender una lengua extranjera; en este caso el inglés. Ésta ha sido identificada por diferentes estudios que muestran, en general, que a mayor motivación, mayor logro de aprendizajes esperados (Gardner y Lambert, 1972; Gardner y MacIntyre, 1991; Cohen y Dörnyei, 2002). Por esto, es importante describir la motivación del alumnado para que tanto autoridades encargadas de las políticas educativas como el profesorado puedan reflexionar sobre estrategias educativas para que el alumnado pueda alcanzar el logro de los aprendizajes.

El presente artículo surge de un estudio a mayor escala que pretende describir la motivación con el Sistema Motivacional del Yo en más de 600 estudiantes de bachillerato, por primera vez en México, por lo que este trabajo reporta sólo la exploración del modelo teórico con alumnado de licenciatura en la última prueba del pilotaje para la construcción del instrumento empleado. En la última fase de pilotaje, este instrumento se aplicó a una muestra de 57 estudiantes de la licenciatura de Diseño y Comunicación Visual de la Facultad de Artes y Diseño de la UNAM donde según el plan de estudios de 8 semestres, inglés es una asignatura curricular obligatoria en cada semestre. Esta fase tuvo como objetivo realizar los últimos ajustes al instrumento, recabar los tiempos de respuesta del cuestionario y revisar la calidad de respuesta del sistema automatizado en línea. A pesar de ser una última fase de prueba de tres que se realizaron, los resultados muestran un alto grado de confiabilidad, consistencia interna y resultados significativos.

En México existen pocos estudios de corte cuantitativo que traten de describir la motivación del alumnado para aprender inglés con el Sistema Motivacional del Yo para L2. Dörnyei (2009) propuso este modelo como una corrección y un avance de modelos teóricos previos. Los modelos actuales que describen la motivación se han construido a partir de estudios empíricos por lo que este modelo ha pasado por diferentes pruebas y se ha validado en diferentes contextos donde el inglés es principalmente lengua extranjera o lengua segunda. Su característica principal es observar a la motivación, incluir más aspectos que afectan a la motivación como la experiencia de aprendizaje, así como sus actitudes;

\section{LA MOTIVACIÓN EN L2/LE}

El éxito de un estudiante para aprender una lengua extranjera estriba en gran parte de la motivación. A partir de los estudios de las últimas décadas, se han elaborado una gran cantidad de investigaciones con respecto a la motivación. En los trabajos más recientes se ha estudiado sobre la naturaleza de la motivación en L2/LE (segunda lengua/lengua extranjera), su relación con factores externos como el bienestar socioeconómico, la influencia social, la asimilación, el utilitarismo, entre otros; así como los factores internos como el nivel de inglés, el autoconcepto, la disposición para hablar, entre otros aspectos. Por lo anterior, se han desarrollado diferentes modelos alrededor de este constructo. Jaclyn Bernard (2010) indica que existen diferentes modelos teóricos y categorizaciones que ayudan al entendimiento académico de la motivación para aprender una lengua segunda/extranjera. Entre estas teorías se encuentran the Theory of Integrative Motivation, o la teoría integradora de la motivación, incorporada en el movimiento psicosocial; además, Self Determination Theory, o Teoría de la autodeterminación, y sus extensiones como la motivación extrínseca e intrínseca.

El Sistema Motivacional del Yo para lengua extranjera o segunda lengua, Motivational Self System, porpuesto por Dörnyei (Ellis, 2015) considera que el principio que subyace en la motivación no es la identificación del aprendiente con los hablantes de la lengua meta, sino con las futuras versiones del yo, es decir, las posibles variantes de uno mismo funcionan como autoguías que marcan lineamientos y objetivos que el aprendiente espera alcanzar. Dörnyei propuso que las autoimágenes del aprendiente como hablante de una segunda lengua están parcialmente basadas en experiencias con la comunidad de hablantes de la lengua meta y parcialmente basadas en la imaginación. De la misma forma, esta teoría da un lugar importante a las condiciones del ambiente de aprendizaje. Puede afirmarse que este modelo interconecta tres teorías: la teoría del Yo, el modelo psico-social y la teoría de la autodeterminación.

Existen tres componentes principales de la teoría de Dörnyei que explican el constructo de la motivación para L2/LE (Dörnyei, 1994; Root, 1999; Lamb, 2012; Ellis, 2015):

- El Yo ideal de L2 (L2 Ideal Self). Refiere la visión propia y personal del aprendiente de la lengua que le gustaría adquirir. Al observar una discrepancia entre la visión futura y la visión actual, el alumnado se verá motivado a mejorar su competencia para convertirse en un hablante con mejores habilidades.

- El Yo necesario de L2 (Ought-to L2 Self). Este componente contiene atributos con los que el aprendiente considera que debe ser y debe poseer para alcanzar sus propias expectativas. Este componente no representa las aspiraciones propias sino de otros grupos de personas como tutores, autoridades como profesores; todas éstas se encuentran ya internalizadas. Se relaciona con motivos instrumentales del tipo de prevención.

- La experiencia de aprendizaje. En este componente, se hallan aspectos relacionados con la situación de aprendizaje y su actitud hacia ella, por ejemplo, el profesorado, el currículo, las amistades, las estrategias de enseñanza, entre otros. 
Con el apoyo de este modelo, se han desarrollado diferentes estudios empíricos en diferentes partes del mundo para validar el modelo teórico y sus instrumentos de recolección de datos. Dörnyei, Csizér y Németh (2006) utilizaron por primera vez El Sistema Motivacional del Yo de L2 para realizar un estudio a nivel nacional en Hungría y determinar el grado de motivación de aprendientes de inglés en la década de los años 90. Se realizaron encuestas a estudiantes con características muy similares en 1993, 1999 y 2004. Asimismo, You y Dörnyei (2015) completaron un estudio a gran escala de corte transversal en el cual se describió la disposición de alumnado en China para aprender inglés en diferentes contextos; el estudio fue realizado de forma estratificada en secundarias y universidades de diferentes regiones de China a aproximadamente 10,000 estudiantes. Martin Lamb (2012) analizó la motivación para aprender inglés de estudiantes en Indonesia a nivel de preparatoria en tres contextos: ciudades, pueblos y el ambiente rural. Estos estudios utilizaron el modelo propuesto por Dörnyei así como su batería de preguntas.

Con este modelo, se concibieron dos tipos de dimensiones (Dörnyei, 2005, 2009) en la instrumentalidad que tradicionalmente se usa: instrumentalización-promoción e instrumentalización-prevención. El primero refiere a motivos instrumentales relacionados con el aprender la lengua, por ejemplo, para alcanzar un desarrollo profesional; mientras que el segundo, con el hecho de evitar un resultado negativo como estudiar inglés para no reprobar la asignatura o decepcionar a alguien como los padres. Por lo tanto, podemos decir que existe una relación de la instrumentalización-promoción con el Yo Ideal de L2 y la instrumentalización-prevención con el Yo Necesario de L2.

\section{METODOLOGÍA}

La metodología empleada para este estudio fue de tipo cuantitativo con un alcance exploratorio. Se llevó a cabo con el apoyo de un cuestionario aplicado en el semestre 2019-1 a estudiantes voluntarios que aceptaron participar de forma anónima al final del periodo.

\section{Participantes}

En este estudio participaron 65 estudiantes de inglés del tercer semestre de la Licenciatura de Diseño y Comunicación Visual de la Facultad de Artes y Diseño de la UNAM. Sin embargo, sólo 57 cuestionarios cumplieron con los criterios de validez o completaron el cuestionario correctamente para ser tomados en cuenta. Inglés es una asignatura curricular en el plan de estudios de la licenciatura y se imparte en los 8 semestres que dura el plan.

El perfil sociodemográfico de los estudiantes es muy heterogéneo ya que provienen de diferentes partes de la Ciudad de México y su zona metropolitana. Cabe mencionar que la Facultad de Artes y Diseño se encuentra al sur de la Ciudad de México en la Alcaldía de Xochimilco, se ofertan tres licenciaturas, asimismo, se encarga de varios posgrados (maestría y doctorado) en artes y diseño, cuenta con departamento de educación continua que imparte talleres y diplomados.

Del alumnado $(\mathrm{n}=57)$, participaron 42 mujeres y 15 hombres cuya edad se encuentra entre los 18 y 25 años. Al final del semestre, se les invitó a los estudiantes a colaborar en la investigación por lo que se les compartió el objetivo del trabajo y la relevancia del proyecto.

\section{Instrumento y pilotaje}

Se diseñó un cuestionario para determinar el grado de motivación del alumnado a partir de instrumentos ya validados y confiables adaptados de los propuestos por Dörnyei (Dörnyei, 2009; Lamb, 2012; You y Dörnyei, 2016) para caracterizar la motivación en sus diferentes investigaciones y otros trabajos bajo este mismo modelo.

El cuestionario contó con 67 reactivos organizados de la siguiente forma:

$$
\begin{aligned}
& \text { - } \quad \text { Motivación para aprender inglés (53). } \\
& \text { - } \quad \text { Perfil académico (8) y sociodemográfico (6). }
\end{aligned}
$$

A continuación, se pueden observar las dimensiones que evaluó el instrumento.

\section{FUTUROS YOS}

- Grado de motivación (comportamiento motivado del aprendizaje)

- Yo ideal de L2

- Yo necesario de L2

INFLUCENCIA SOCIAL EN L2

- Influencia familiar / tutores

- Influencia de pares

- Influencia del profesorado

\section{OTROS FACTORES QUE INFLUYEN EN LA MOTIVACIÓN}

- $\quad$ Instrumentalización - Prevención
- $\quad$ Instrumentalización - promoción
escuela/Actitudes hacia el
aprendizaje de inglés
Miedo hacia la asimilación
- $\quad$ Etnocentrismo

La segunda parte del cuestionario, perfil académico y el perfil sociodemográfico, recabó información relacionada con sus estudios, edad, sexo y nivel percibido de inglés. 


\section{Aplicación del cuestionario}

La versión final del cuestionario fue aplicada en noviembre de 2018, al final del semestre 2019-1; se invitó al estudiantado de inglés semestre 3 a participar de forma anónima y voluntaria; contestaron el cuestionario de forma digital con el apoyo del sistema LimeSurvey montado en un servidor Linux. 65 colaboradores aceptaron realizar el cuestionario; les tomó un promedio 15 minutos en contestar. Sin embargo, 57 cuestionarios cumplieron con los estándares.

\section{Análisis de datos}

El análisis de datos fue realizado con el programa SPSS versión 25 para obtener los principales estadísticos descriptivos de tendencia central (mínimos, máximos, media y desviación estándar); así como el Alfa de Cronbach.

\section{RESULTADOS}

\section{Instrumento}

Algunos de los coeficientes del Alfa de Cronbach superaron 0.8 o estuvieron cercanos a alcanzarlo. Sin embargo, hubo también constructos con coeficientes muy bajo (0.592). Según Dörnyei (2003 y 2010), en las investigaciones de lengua segunda y lengua extranjera es común no alcanzar 0.8 en el Alfa "[b]ecause of the complexity of the second language acquisition process, L2 researchers typically want to measure many different areas in one questionnaire, and therefore cannot use very long scales (or the completion of the questionnaire would take several hours)" (Dörnyei, 2003). No obstante, el Alfa muestra un grado aceptable de consistencia interna del instrumento para la investigación.

Los constructos que mostraron un coeficiente más alto fueron:

- La experiencia de aprendizaje de L2 en la escuela/Actitudes hacia el aprendizaje de inglés con 0.820

- Influencia del profesorado con 0.82

Mientras que el constructo más bajo fue:

- Instrumentalización - Promoción con 0.592

\section{La motivación y sus componentes}

La siguiente tabla muestra la media, el error y la desviación estándar de los constructos ordenados de mayor a menor. El primer lugar ocupa la dimensión de Instrumentalización-Promoción con una media de 5.515 y desviación estándar de 0.488; en segundo lugar se encuentra el Yo ideal de L2 con una media de 5.286 y DE=0.545; ambos exponen que el alumnado de esta muestra está consciente de la importancia del inglés y las ventajas que podrían obtener al alcanzar un nivel avanzado en el dominio de la lengua.
Tabla 1. Descriptivos generales de las variables

\begin{tabular}{|c|c|c|c|}
\hline \multirow[t]{2}{*}{ Aspecto } & \multicolumn{2}{|c|}{ Media } & \multirow{2}{*}{$\begin{array}{c}\mathrm{DE} \\
\text { Estadístico }\end{array}$} \\
\hline & Estadístico & $\begin{array}{l}\text { Error } \\
\text { estándar }\end{array}$ & \\
\hline Instrumentalización - promoción & 5.5158 & 0.0646 & 0.4883 \\
\hline Yo ideal de L2 & 5.2865 & 0.07223 & 0.54532 \\
\hline Influencia del profesor & 4.9474 & 0.1118 & 0.8446 \\
\hline $\begin{array}{l}\text { Comportamiento motivado del } \\
\text { aprendizaje }\end{array}$ & 4.8333 & 0.08938 & 0.6748 \\
\hline Influencia de pares & 4.7661 & 0.04152 & 1.09068 \\
\hline Influencia familiar / padres & 4.6486 & 0.1042 & 0.7867 \\
\hline Yo necesario de L2 & 4.4678 & 0.1074 & 0.8112 \\
\hline $\begin{array}{l}\text { La experiencia de aprendizaje de L2 en la } \\
\text { escuela/Actitudes hacia el aprendizaje de } \\
\text { inglés }\end{array}$ & 4.3421 & 0.12029 & 0.9082 \\
\hline Instrumentalización - Prevención & 4.2237 & 0.12048 & 0.9096 \\
\hline Etnocentrismo & 3.1023 & 0.1050 & 0.7929 \\
\hline Miedo hacia la asimilación & 2.5746 & 0.1428 & 1.0783 \\
\hline
\end{tabular}

La tercera dimensión relativamente alta es la Influencia del profesorado con una media de 4.947 y $\mathrm{DE}=0.844$; el cuarto lugar es el comportamiento motivado del aprendizaje con una media de 4.833 y $\mathrm{DE}=0.674$; la quinta es la influencia de pares con una media de 4.7661 y DE=1.09068; en la sexta posición se puede observar la influencia familiar con una media de 4.6486 con $\mathrm{DE}=0.7867$; la séptima posición, Yo necesario de L2 con una media de 4.4678 y DE=0.8112; la séptima, la Experiencia de aprendizaje de L2 con una media de 4.3421 y DE=0.9082.

Por otro lado, los extremos, los de más baja escala, etnocentrismo con una media de $\mathrm{x}=3.1023$ y $\mathrm{DE}=0.792$; así como el miedo a la asimilación con una media de 2.574 y $\mathrm{DE}=1.078$ cuyo significado refiere a la apertura ideológica y al sincretismo.

\section{DISCUSIÓN}

El objetivo de este trabajo fue mostrar los primeros resultados de la fase de prueba de un estudio de mayor amplitud (más de 600 participantes de preparatoria) para describir la motivación para aprender inglés con el Sistema Motivacional del Yo para L2 en el estudiantado de licenciatura cuya asignatura de inglés es obligatoria en el plan de estudios en los 8 semestres. Se utilizaron los tres componentes principales del Sistema Motivacional del Yo (Yo Ideal de L2, Yo Necesario y Experiencia de Clase); asimismo, otras dimensiones comúnmente relacionadas con la motivación como son la instrumentalización (prevención y promoción), la influencia de pares, familiares y del profesorado, así como el etnocentrismo y el miedo a la asimilación para medir la apertura ideológica a la otredad y el cambio.

En general, gracias a los resultados se puede observar una fuerte influencia entre el Yo Ideal de L2, la influencia del profesorado 
y el comportamiento motivado al aprendizaje como lo reportan otros estudios (Kormos, Kiddle y Csizér, 2011; Lamb, 2012; You y Dörnyei, 2014).

Como era de esperarse, la instrumentalización-promoción $(x=5.515, D E=0.488)$ es el coeficiente que sobresale al ser la primera dimensión, le sigue el Yo Ideal de L2 ( $\mathrm{x}=5.286$, $\mathrm{DE}=0.545$ ) que exceden por mucho el punto medio (3.5); el primero refiere a la utilidad instrumental del inglés, es decir, lo conciben como una herramienta para proporcionar el acceso a mejores oportunidades laborales o educativas. Podríamos decir que en general los participantes conciben al inglés como una herramienta para facilitarles el acceso a mejores oportunidades laborales o educativas. Es una de las principales dimensiones y, comúnmente, la más estudiada. El segundo implica que los participantes de esta muestra están conscientes de la importancia del inglés y las ventajas que podrían obtener al alcanzar un nivel avanzado en el dominio de la lengua. Los puntajes del Yo ideal de L2 ( $\mathrm{x}=5.286, \mathrm{DE}=0.545)$ exponen que la mayoría de las y los estudiantes poseen una imagen futura como hablantes competentes de inglés capaces de interactuar y comunicarse efectivamente en diferentes contextos en el extranjero como en México. Se imaginan competentes para entablar comunicación con personas provenientes de diferentes partes del mundo. Asimismo, se observan en un futuro utilizando el inglés en sus estudios universitarios como profesionales.

El tercer elemento con un coeficiente alto es la influencia del profesorado de $(\mathrm{x}=4.947$ y $\mathrm{DE}=0.844)$. Esto puede apuntar a que el rol del profesorado es crucial en transformar las actitudes positivas del inglés en un esfuerzo real de aprendizaje; o, en caso contrario, a influir en representaciones negativas hacia la lengua. Aunque la desviación estándar cercana al punto, que describe una mayor dispersión entre las y los participantes, se puede observar un peso relevante de esta dimensión en la motivación de las y los participantes de esta muestra. Sin embargo, existen pocos estudios que estudien los efectos de la práctica docente y las actitudes del profesorado en la clase. Por lo anterior, se puede decir que la acción docente y las actitudes del profesorado en la clase tienen una gran influencia en si el estudiantado lleva a cabo acciones para seguir aprendiendo o detener sus actividades.

Esto revela un efecto considerable en la percepción del alumnado por parte de la acción docente y las estrategias didácticas instrumentadas; asimismo, se puede percibir un comportamiento motivado al aprendizaje alto, es decir, el alumnado de esta muestra realiza actividades académicas por su cuenta, así como aprovecha el tiempo para aprender inglés como lengua extranjera.

Las siguientes dimensiones son la influencia de pares y la influencia familiar lo que involucra la importancia del contexto familiar como de las amistades en la motivación. Estas influencias pueden llegar a ser tan importante como la influencia del profesorado. Generan representaciones positivas o negativas sobre la lengua, sus hablantes y su cultura lo que puede influir directamente en la toma de decisión para seguir estudiando o dejar de hacerlo.

Otro elemento que sobresale es la experiencia de aprendizaje. Aquí podemos afirmar que entre el estudiantado se hallan aspectos positivos relacionados con su situación de aprendizaje y su actitud hacia ella durante su trayectoria. Esto puedo implicar:

La dimensión del Yo Necesario de L2 muestra una escala superior a la media pero de las medias más bajas en este estudio. A diferencia de otros estudios (Lamb, 2012; You y Dörnyei, 2014) donde el Yo Necesario de L2 muestra uno de los puntajes más bajos, en el presente estudio tiene un puntaje alto en general. Como el Yo Necesario muestra principalmente los aspectos de la motivación extrínseca, así como aspiraciones de grupos externos como profesores, tutores, gobierno, entre otros, al ser inglés una asignatura curricular y obligatoria, se puede presumir que existe la necesidad de aprobar la asignatura para completar los créditos por lo que les implicaría un mayor esfuerzo.

De la misma manera ocurre con la instrumentalizaciónprevención en la tendencia en los subgrupos. Al igual que el Yo Necesario, la instrumentalización-prevención contiene un aspecto de necesidad y obligatoriedad lo que la agrupa con pensamientos, ideas y motivos relacionados con la evasión de consecuencias negativas como estudiar para no reprobar el curso de inglés, para no decepcionar a sus tutores o un ser querido. Esto puede sugerir que el alumnado muestra una necesidad de no reprobar el curso y obtener una buena calificación para cumplir con sus créditos por lo que les implica un mayor esfuerzo y trabajo para obtener una mejor calificación. A diferencia de otras investigaciones cuyo coeficiente es bajo, esta muestra despliega un mayor coeficiente que podría indicar la sensación de obligatoriedad percibida por las y los participantes para acreditar la asignatura.

La dimensión relacionada con el etnocentrismo $(x=3.1023 \mathrm{y}$ $\mathrm{DE}=0.792$ ). Este coeficiente indica una representación baja del estudiantado, aunque considerable, de superioridad cultural propia frente a una extranjera y su nacionalismo. Por el contrario, un nivel alto de este coeficiente en el estudiantado podría ser un reto de aprendizaje de una L2 ya que la representación de la cultura extranjera tendría su valor a partir de la cultura propia como criterio exclusivo para interpretar y valorar la cultura, así como comportamientos de la cultura extranjera.

Por último, el miedo a la asimilación (2.574 y $\mathrm{DE}=1.078)$ representa el grado de aceptación de elementos culturales extranjeros hasta integrarlos en sus comportamientos, valores o creencias. El coeficiente bajo podría ser una ventaja en el aprendizaje de inglés ya que es más probable que el estudiantado sea más capaz de aceptar comportamientos culturales extranjeros incluso al punto de adoptarlos. No 
obstante, el miedo a la asimilación muestra una desviación estándar mayor al punto, lo que describe una mayor dispersión entre los individuos.

Estas dos últimas dimensiones presentan una mayor apertura ideológica, cultural y étnica lo que permite una mejor adaptación y aproximación a las culturas de habla inglesa; Entonces, los participantes muestran una mayor capacidad de tener una mente abierta a la otredad, a la cultura e idiomas extranjeros, así como al intercambio de ideas y pensamientos, elementos indispensables al aprender un nuevo idioma, es decir, a las diversas representaciones de la realidad que los grupos sociales pueden poseer.

\section{CONCLUSIÓN}

Los altos puntajes en Instrumentalización-Promoción y Yo ideal de L2 sugieren que los y las participantes de esta muestra están conscientes de la importancia del inglés y las ventajas que podrían obtener al alcanzar un nivel avanzado en el dominio de la lengua; entienden al inglés como una herramienta para proporcionarles el acceso a mejores oportunidades laborales o educativas. De la misma manera, la influencia del profesorado puede percibirse como un factor importante el en impulso en la motivación ya que la acción docente y las estrategias didácticas instrumentadas podrían influir en el impulso para seguir aprendiendo por lo que podríamos afirmar también que este constructo tiene una relación con la experiencia de clase, un punto necesario a estudiar en próximos estudios. Asimismo, los bajos puntajes de etnocentrismo y miedo a la asimilación exponen una mayor apertura ideológica apoyando a una mejor adaptación y aproximación a las culturas de habla inglesa. Entonces, se puede advertir una mayor apertura a la otredad, a la cultura e idiomas extranjeros.

Los resultados de este estudio muestran una relación muy cercana con estudios anteriormente mencionados en cuestión la Instrumentalización-Promoción del inglés, el Yo Ideal de L2, el comportamiento motivado y la influencia del profesorado. Mencionamos la necesidad de analizar el grado de correlación entre constructos como la experiencia de aprendizaje, la influencia del profesorado, el comportamiento motivado, el Yo Ideal y el Yo Necesario de L2. Sabemos que existe una relación muy fuerte entre la motivación y los logros de los aprendizajes (Gardner y Lambert, 1972; Gardner y MacIntyre, 1991; Cohen y Dörnyei, 2002) por lo que es importante realizar estudios que analicen la correlación entre la motivación y estas variables para que los diseñadores instruccionales, profesores, diseñadores curriculares, asesores, monitores, entre otros, adviertan que son elemento clave en la forma en cómo propician un ambiente de aprendizaje adecuado y esto repercute en la motivación por lo que la formación docente puede llegar a ser un elemento clave al que no se le había puesto atención. Se escucha que la motivación depende del alumnado; no obstante, no se ha estudiado profundamente el grado de influencia del profesorado y la experiencia de clase como catalizadores en el logro de los aprendizajes.

\section{REFERENCIAS}

[1] Boyatzis, R. E., y Akrivou, K. (2006). The ideal self as the driver of intentional change. Journal of Management Development, 25, 624-642.

[2] Bursal11, N. y Öz, H. (2017). The Relationship between Ideal L2 Self and Willingness to Communicate Inside the Classroom. En International Journal of Higher Education, 6(4), pp. 229-239. doi:10.5430/ijhe.v6n4p229

[3] Cohen, A. D., y Dörnyei, Z. (2002). Focus on the language learner: Motivation, styles, and strategies. In N. Schmitt (Ed.) An introduction to applied linguistics (pp. 170-190). London, England: Edward Arnold.

[4] Dörnyei, Z. (1994). Motivation and Motivating in the Foreign Language Classroom. The Modern Language Journal, 78(3), pp. 273-284. Recuperado de http://www.jstor.org/stable/330107

[5] Dörnyei, Z. (2001) Motivational Strategies in the Language Classroom. Cambrdige: Cambridge University Press.

[6] Dörnyei, Z. (2003). Questionnaires in Second Language Research. Construction, Administration, and Processing. Londres: Lawrence Erlbaun.

[7] Dörnyei, Z. (2005). The Psychology of the Language Learner: Individual Differences in Second Language Acquisition. Mahwah, NJ: Lawrence Erlbaum Associates.

[8] Dörnyei, Z. (2009). The L2 Motivational Self System. En Z. Dörnyei y E. Ushioda (Eds), Motivation, language identity and the L2 self (pp. 9-42). Bristol: Multilingual Matters.Bonal-Ruiz R, Cascaret-Soto X. Reflexión y debate ¿Automanejo, autocuidado o autocontrol en enfermedades crónicas? Acercamiento a su análisis e interpretación. MEDISAN. 2009; 13(1): 1-10.

[9] Dörnyei, Z. (2010). Questionnaires in Second Language Research: Construction, Administration, and Processing, 2nd ed. Routledge.

[10] Dörnyei, Z., Csizér, K. and Németh, N. (2006). Motivation, Language Attitudes and Globalisation. Clevedon: Multilingual Matters Ltd.

[11] Ellis, R. (2015). Understanding Second Language Acquisition. 2 ed. Londres: Oxford University Press.

[12] Gardner, R. C., y Lambert, W. E. (1972). Attitudes and motivation in second language learning. Rowley, MA: Newbury House.

[13] Kormos, J., Kiddle, T. y Csizér, K. (211). Systems of Goals, Attitudes, and Self-related Beliefs in SecondLanguage-Learning Motivation. En Applied Linguistics, 32 (5), 495-516 pp., https://doi.org/10.1093/applin/amr019

[14] Lamb, M. (2012). A Self System Perspective on Young Adolescents' Motivation to Learn English in Urban and Rural Settings. En Language Learning Research Club, 9971023 pp. Universidad de Michigan.

[15] Pallant, J. (2007). SPSS survival manual (3rd ed.). New York: Open University Press. 
[16] Root, E. (1999). Motivation and Learning Strategies in a Foreign Language Setting. CARLA. Minesota: Universidad de Minesota.

[17] Tremblay, P. y Gardner, R. (1995). Expanding the Motivation Construct in Language Learning. The Modern Language Journal, 79(4), pp. 505-518. Recuperado de The Modern Language Journal.

[18] You, J. y Dörnyei, Z. (2014). Language Learning Motivation in China: Results of a Large-Scale Stratified Survey. Applied Linguistics 2016, 495-519. Oxford University Press.

[19] You, J. y Dörnyei, Z. (2016). Language Learning Motivation in China: Results of a Large-Scale Stratified Survey. Applied Linguistics 2016, 495-519. Oxford University Press. 